\title{
Protein Structure and Function Analysis Method of Aminoacyl-tRNA Synthetase Cofactor and Biotinylation Effect: Journal Review
}

\author{
Restu Nugraha ${ }^{1,2^{*}}$, Chien-Chia Wang ${ }^{2}$, Widodo ${ }^{1}$ \\ ${ }^{1}$ Department of Biology, Faculty of Mathematics and Natural Science, University of Brawijaya, Malang, Indonesia \\ 2Department of Life Sciences, National Central University, Taoyuan, Taiwan
}

\begin{abstract}
Protein has been known as an important macromolecule which has a vital role among the living organism. One of the most interesting protein is Arc1p, which is a yeast-specific tRNA-binding protein. Arc1p is a unique protein that has the ability to form a ternary complex with glutamyl-tRNA synthetase (GluRS ${ }_{c}$ ) and methionyl-tRNA synthetase (MetRS) in the cytoplasm. This complex can significantly enhance the aminoacylation efficiency of these two aaRSs to their respective cognate tRNAs. Recently, it was found that Arc1p can be biotinylated via post-translational modification at Lys86 (K86) in the N-domain. Here, we try to figure it out what kind of method that will help to create some clear information both in structure and function of this protein, when mutations occur inside of the K86 site within SSKD motifs of Arc1p. Several methods to better understanding obviously about protein characteristics comprises protein structural analysis; such as gel mobility shift assay, CD Spectroscopy, and limited proteolysis; protein functional analysis, and in silico modeling.
\end{abstract}

Keywords: Arc1p, biotinylation, function, in silico, structure.

\section{INTRODUCTION}

Protein is an important macromolecule which has an indispensable role in the widely living organism. Analysis of protein became a very important step in biochemistry and biomolecular study. Extensive application from this analysis will be more helpful to understand both structure and function. For example, is protein Arc1. Arc1p (Aminoacyl-tRNA Synthetase Cofactor 1) is a yeast-specific tRNA-binding protein that has the ability to make a ternary complex with glutamyl-tRNA synthetase (GluRS $c$ ) and methionyl-tRNA synthetase (MetRS) in the cytoplasm. This complex can significantly enhance the aminoacylation efficiency of these two aaRSs to its cognate tRNAs. Recently, Arc1p can be biotinylated via post-translational modification at Lys86 (K86) in the N-domain. Lysine itself commonly plays an important role in the structure [1]. Identification of Arc1p as a novel biotinylated yeast protein became very intriguing to find since Arc1p is not known to be involved in any carboxylation reaction (carboxylation, decarboxylation, or transcarboxylation reactions) [2].

Furthermore, in Arc1p a lack of sequence similarity was reviewed between the biotin binding site and the highly conserved biotin

\footnotetext{
* Correspondence address:

Restu Nugraha

Email : restunugraha10@gmail.com

Address : Dept. Biology, University of Brawijaya, Veteran Malang, Malang, 65154
}

binding consensus sequence of known carboxylases, AMKM [3]. Mostly biotinylated lysine residues are positioned within this consensus. However, Arc1p may still be biotinylated in vivo. One interesting thing is that biotinylation of Arc1p was performed by the same enzyme which also catalyzes some yeast carboxylases, Bpl1p. SSKD in Arc1p may represent a secondary biotinylation site for Bpl1p [3]. In this review, we try to figure it out what kind of method that will help to create some clear information both in structure and function of this protein, when mutations occur inside of the K86 site within SSKD motifs of Arc1p.

\section{PROTEIN STRUCTURE ANALYSIS METHOD Streptavidin-based Gel Mobility Shift Assay}

Gel mobility shift assay is a usual and common tool in molecular biology to check characteristic of the protein. In this case, Arc1p known has biotinylation activity [3]. Thus, in order to measure the relative biotinylation levels of Arc1p variants from WT and yeast mutants, a streptavidin-based gel mobility shift assay was determined [4]. This phenomenon was based on due to the almost irreversible binding of biotin with streptavidin $\left(K_{\mathrm{d}}=10^{-14} \mathrm{M}\right)$ [5]. This binding is one of the strongest interactions between noncovalent molecules. Highest known affinity in nature between these two molecules has been largely applied as a powerful tool for diagnostic purposes [6].

Besides streptavidin, avidin also has the ability to binding with biotin. Moreover, there 
was research of 3D structures of both avidin and streptavidin to compare their binding properties. This research exhibit that both proteins have a conserved tyrosine-containing stretch (Fig. 1) $[6,7]$ The difference between avidin and streptavidin is the lengths of the loops that happened when binding of biotin, 36-44 in avidin and 45-50 in streptavidin, respectively. As consequences, the longer loop of avidin causes tighter closure of the occupied binding site [6]. Recently reported that avidin has a tighter binding of biotin than streptavidin ( $K_{d}=6 \times 10^{-16}$ and $4 \times 10^{-14} \mathrm{M}$, respectively) [8].

$$
\begin{array}{ll}
\text { AVIDIN } & \text { Thr }- \text { Gly }- \text { Thr }- \text { Tyr }-\| l e-\text { Thr }- \text { Ala }- \text { Val } \\
& 40 \\
\text { STREPTAVIDIN } & \text { Thr - Gly - Thr }- \text { Tyr }- \text { Glu-Ser-Ala - Val }
\end{array}
$$

Figure 1. Conserved sequences of avidin and streptavidin tyrosine-containing stretches [7]

\section{Circular Dichroism (CD) Spectroscopy}

Circular Dichroism (CD) Spectroscopy is well known as a common method in order to study and analyze the secondary structure or conformation changes of macromolecules, in which sensitive to its environment, temperature, $\mathrm{pH}$, or interaction with other molecules. An important concept of CD spectroscopy was that this is a result of the interaction of polarized light with chiral molecules since most biological molecules are chiral (Fig. 2). The best example is 19 of the 20 common amino acids that form proteins are themselves chiral, along with the higher structures of proteins, like DNA and RNA [9]. Recently, most widely applied of circular dichroism are for study the secondary structure of proteins, such as the $\alpha$-helix and the $\beta$ sheet (Fig. 3).

Moreover, this method allows us to explore deeper the higher order structures of chiral macromolecules including proteins and DNA. Importantly, in CD spectrum, the structure of a DNA molecule or protein is not a total of the CD spectra from the individual residues or bases but is extremely influenced by the 3-dimension structure of the macromolecule itself. This could be because each structure from a macromolecule has a specific circular dichroism characteristic, and allow to identify structural elements at once the structure changes of chiral macromolecules. Principally, the $C D$ is an inference from the interaction among polarized light with chiral molecules [9].
In this case, CD Spectroscopy was used in order to investigate whether biotinylation affects the secondary structure of Arc1p variants. Using purified Arc1p, the secondary structure changes of Arc1p, eventually revealed. Protein structure can be determined by CD Spectroscopy in the far UV, which is an $\alpha$-helix has the negative band at 222 and $208 \mathrm{~nm}$, and $\beta$-sheet has a negative band at $218 \mathrm{~nm}$. Also, CD Spectroscopy conducted to compare the thermal stabilities of Arc1p variants [4].

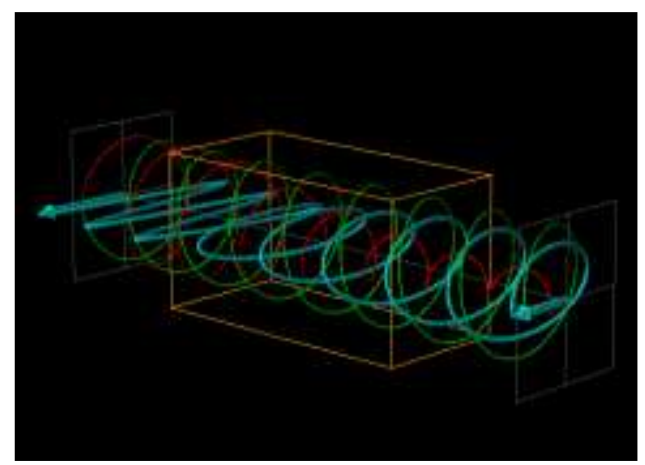

Figure 2. The principle of circular dichroism (orange cuboid represents the sample) [9].
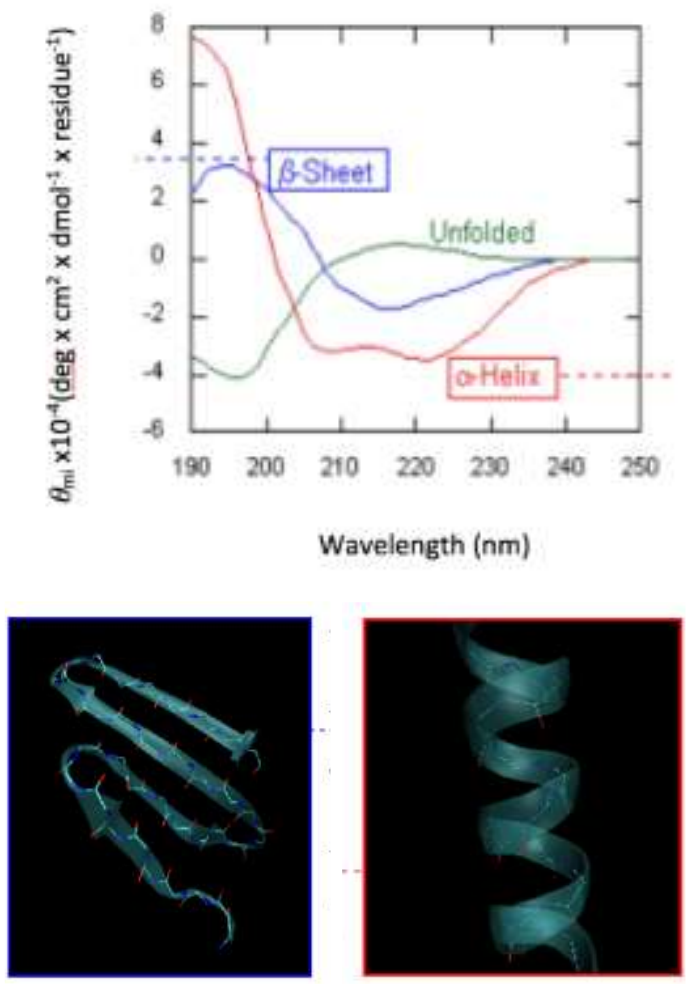

Figure 3. The secondary structure conformation and the $C D$ spectra of protein structural elements. Upper is the associated CD spectra. Bottom left (blue outline) is a $\beta$-sheet and the bottom right (red outline) is an $\alpha$-helix [9]. 


\section{Limited Proteolysis}

Limited Proteolysis was used to check and analyze structure conformational changes of the protein $[10,11]$. The main concept of this method is the incubation of protein with one or several types of protease at various time. Subsequently, this enzyme will be cut at recognition sites inside the protein (Fig. 4) [12,13]. Proteolysis of a protein substrate specifically occur only if the polypeptide chain has the ability to bind and adapt to the specific stereochemistry of protease's active site [14]. We can change some variables, such as type of protease, dilution of protease, temperature and time of incubation. After digestion by protease, identification of cleavage products could be identifying by SDSPAGE. Digestion of full-length protein can be detected by the lower molecular weight bands, together with intensity and appearance of bands can be observed [13].

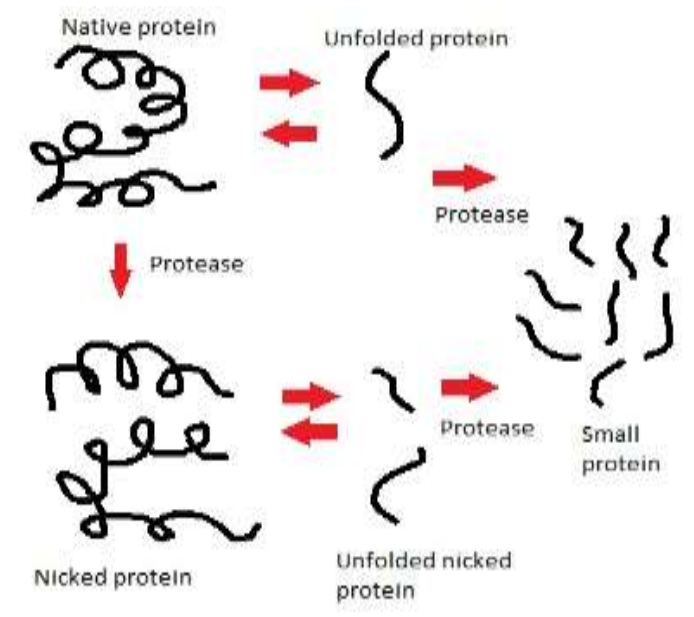

Figure 4. Principle of Limited Proteolysis [17]

Various application of limited proteolysis has been discovered. This method was very useful in protein study such as crystallization studies, or in situ proteolysis for protein crystallization and structure determination; like to expect the presence of stable sub-domains [12]. Limited proteolysis also can be used as a surface probe for membrane proteins [15]. Besides, limited proteolysis also able to detect binding between one protein to another, in a way by running two identical sets of proteolysis experiments, with and without the putative target. The expected result will exhibit by the rate of digestion of the protein. When it is slowed by the addition of the putative target, one can take finding that they interact with each other $[12,16]$.

In the case of Arc1p, limited proteolysis can be used in order to test whether biotinylation will alter the structural flexibility of Arc1p. Limited proteolysis with Arc1p/trypsin using is a ratio of $1,000: 1$ and conducted at 30 and $37^{\circ} \mathrm{C}$. They were using $0,8,16$, and 16 minutes of time [3]. After the treatment of trypsin, the result will be obtained which Arc1p is more flexible in term of structure than the others.

\section{PROTEIN FUNCTION ANALYSIS METHOD Complementation Assay}

Arc1p has become one of the most intriguing research topics in recent years. Arc1p is an auxiliary protein of the MARS complex, which also consists of two aaRSs, GluRS and MetRS. This protein is also known as a homolog of human p43. Arc1p has a key role in some cellular activities, such as in multiple cellular pathways, tRNA channel, and nucleocytoplasmic transport $[2,18]$.

Complementation assay generally used to test the rescue activity of knock out strain. This method could be conducted to analyze mitochondrial or cytoplasm activity. Selective medium for cytoplasm and mitochondrial activity were using 5-fluoroorotic acid (5-FOA) and YPG medium, respectively [19]. In the case of Arc1p, complementation assay was held in order to investigate whether biotinylation of Arc1p will affect the activity in vivo. Genes encoding the wild type and mutant of Arc1p were respectively cloned in $\mathrm{pADH}$ vector (high-copy number yeast shuttle vector with a constitutive $A D H$ promoter and a LEU2 marker). Subsequently, the resulting constructs were tested in an $\operatorname{arc1} 1^{-}$yeast strain at $20^{\circ} \mathrm{C}, 30^{\circ} \mathrm{C}$, and $37^{\circ} \mathrm{C}$ respectively in SD/-Leu plates [3]. Using this method, we can conclude whether biotinylation is needed for rescue activity of Arc1p.

\section{IN SILICO MODELING}

There was some method related to structure analysis of protein. One kind of method is in silico modeling. In silico modeling methods are computational-based approaches to analyze and simulate the macromolecules, include protein. In this method we also able to predict and visualize molecule. Recently, in silico modeling is often used in combination in vivo analysis [20]. Moreover, in silico tool also widely used in pharmacology, toxicology, and drugs. For example, this method has been developed to predict all drugs activity and reaction once they get in into the human body or certain organism [21].

In this case, Arc1p can be analyzed by interaction with another molecule, such as GluRS 
and MetRS. As we know, Arc1p is an auxiliary protein of the MARS complex, which also consists of two aaRSs, GluRS and MetRS [2]. Based on a recent experiment, the catalytic efficiency of Arc1p-GluRS increase by 100 -fold for its cognate tRNA $^{\text {Glu }}$ [22]. Using in silico modeling, we allowed to analyzing chemical and biological properties in Arc1p to represents within software tools to predict.

\section{CONCLUSION}

Arc1p defined as yeast-specific tRNA-binding protein and has the primary ability to make a ternary complex with glutamyl-tRNA synthetase (GluRS ) and methionyl-tRNA synthetase (MetRS) in the cytoplasm [3]. This complex's main function is to enhance the aminoacylation efficiency of both, GluRS $c$ and MetRS, for their cognate tRNAs. Specifically, Arc1p acts as a tRNAattracting molecule thus enhancing tRNA availability for these two enzymes $[5,23]$. Although Arc1p lacks a highly conserve biotinylation consensus and does not engage in any biotin-dependent carboxylases reaction, Arc1p still has biotinylation activity, even when modified by Bpl1p in yeast $[3,24]$. One another interesting thing is that Arc1p is only biotinylated by Bpl1p, while other biotin protein ligases, such as HCS in mammals and BirA in E. coli, do not act on Arc1p as a substrate. It has recently been known that a high degree of similarity among biotin binding domains makes broad substrate specificity. Since it is known that biotin protein ligase has the ability not only to biotinylate different in the apoenzyme in the same organism but even came from different organisms, this finding is remarkable. Like in previous reports, the SSKD motif may represent a secondary biotinylation site for yeast Bpl1p [25]. Despite having very vital functions, $\operatorname{arc} 1 \Delta$ mutants are not lethal. This is caused by the operation of a second, exportin Los1p which is required by aminoacylation-independent nuclear tRNA export pathway in yeast [26]. Lethality in yeast only occurred when inactivation of these two genes, ARC1 and LOS1 occurred simultaneously, resulting in no additional tRNA export pathway existing in yeast $[18,23]$.

In this review, we could see some method which usually uses in the study of the structure and function of a protein. Based on the description above, Arc1p is likely to remain one of the interesting research objects in the biomolecular field. Some of the methods described above will open up new knowledge regarding the characteristics of this protein. Subsequently, from the results of the structure and function analysis, may be able to draw conclusions, why Arc1p has very unique roles and characteristics. This may lead to a better understanding of several methods of a complete view related to protein attributes. Thus, our review about some methods in the study of protein characteristics, especially in Arc1p, may only represent a little in fact that nowadays technology and molecular techniques are absolutely developing rapidly.

\section{ACKNOWLEDGMENT}

This research was supported by Ministry of Science and Technology (MOST), Taiwan.

\section{REFERENCES}

[1] Betts, M.J., B.R. Robert. 2003. Amino acid properties and consequences of substitutions. In: Barnes, M.R., I.C. Gray (Eds). Bioinformatics for Geneticists. John Wiley and Sons, Ltd.

[2] Edvardson, S., A. Shaag, O. Kolesnikova, J.M. Gomori, I. Tarassov, T. Einbinder, O. Elpeleg. 2007. Deleterious mutation in the mitochondrial arginyl-transfer RNA synthetase gene is associated with pontocerebellar hypoplasia. Am. J. Hum. Genet. 81. 857-862.

[3] Yao, C.C., C. Chia-Pei, C. Shruti, W. ShaoWin, T. Yi-Kuan, W. Chien-Chia. 2016. Modulating the structure and function of an aminoacyl-tRNA synthetase cofactor by biotinylation. J. Biol. Chem. 291(33), 1710217111.

[4] Nathanson, L., M.P. Deutscher. 2000. Active aminoacyl-tRNA synthetases are present in nuclei as a high molecular weight multienzyme complex. J. Biol. Chem. 275. 31559-31562

[5] Frechin, M., K. Daniel, P.M. Robert, D.B. Hubert, S. Bruno. 2010. Arc1p: Anchoring, routing, coordinating. FEBS Lett. 584. 427433.

[6] Livnah, O., A.B. Edward, W. Meir, L.S. Joel. 1993. Three-dimensional structures of avidin and the streptavidin-biotin complex. Proc. Natl. Acad. Sci. USA. 90. 5076-5080.

[7] Gitlin, G., A.B. Edward, W. Meir. 1990. Studies on the biotin-binding sites of avidin and streptavidin. Biochem. J. 269. 527-530.

[8] Green, N.M. 1990. Avidin and streptavidin. Methods Enzymol. 184. 51-67. 
[9] Dmitry. 1999. An introduction to circular dichroism spectroscopy. Available at: https://www.chem.uci.edu/.

[10] Fontana, A., P.P. de Laureto, V. De Filippis, E. Scaramella, M. Zambonin. 1997. Probing the partly folded states of proteins by limited proteolysis. Folding Design. 2(2). R17-R26.

[11] Hubbard, S.J. 1998. The structural aspects of limited proteolysis of negative proteins. Biochim. Biophys. Acta. 1382(2). 191-206.

[12] Moldoveanu, T., C.M. Hosfield, Z. Jia, J.S. Elce., P.L. Davies. 2001. $\mathrm{Ca}^{2+}$-induced structural changes in rat $m$-calpain revealed by partial proteolysis. Biochim. Biophys. Acta. 1545(1-2). 245-254.

[13] Quevillon-Cheruel, S., B. Collinet, L. Trésaugues, P. Minard, G. Henckes, R. Aufrère, K. Blondeau, C.Z. Zhou, D. Liger, N. Bettache, A. Poupon, I. Aboulfath, N. Leulliot, J. Janin, H. van Tilbeurgh. 2007. Cloning, production, and purification of proteins for a medium-scale structural genomics project. Methods Mol. Biol. 363. 21-37.

[14] Schechter, I., A. Berger. 1967. On the size of the active site in proteases. I. Papain. Biochem. Biophys. Res. Commun. 27. 157162.

[15] Hargrave, P.A., S.L. Fong, J.H. McDowell, M.T. Mas, D.R. Curtis, J.K. Wang, E. Juszczak, D.P. Smith. 1980. The partial primary structure of bovine rhodopsin and its topography in the retinal rod cell disc membrane. Neurochem. Int. 1. 231-244.

[16] Chazin Lab. 2002. Chazin Lab protocol for limited proteolysis. Available at: http://structbio.vanderbilt.edu/chazin/wisd om/labpro/proteolysis.html

[17] Fontana, A., P. de L. Patrizia, S. Barbara, F. Erica, P. Paola, M. Zambonin. 2004. Probing protein structure by limited proteolysis. CRIBI Biotechnology Centre, University of Padua, Padua, Italy.

[18] Ibba, M., D. Soll. 2000. Aminoacyl-tRNA synthesis. Annu. Rev. Biochem. 69. 617-650.

[19] Chang CY., C.I. Chien, C.P. Chang, B.C. Lin, C.C. Wang. 2016. WHEP domain regulates the dynamic structure and activity of Caenorhabditis elegans glycyl-tRNA synthetase. J. Biol. Chem. 291(32). 1656716575

[20] Myatta, Glenn J. et al. 2018. In silico toxicology protocols. Regul. Toxicol. Pharmacol. 96. 1-17.
[21] Pinero, J., I.F. Laura, S. Ferran. 2018. In silico models in drug development: where we are. Curr. Opin. Pharmacol. 42.111-121.

[22] Graindorge, J.S., B. Senger, D. Tritch, G. Simos, F. Fasiolo. 2005. Role of Arc1p in the modulation of yeast glutamyl-tRNA synthetase activity. Biochemistry. 44. 13441352.

[23] Simos, G., A. Sauer, F. Fasiolo, E.C. Hurt. 1998. A conserved domain within Arc1p delivers tRNA to aminoacyl-tRNA synthetases. Mol. Cell. 1. 235-242.

[24] Beckett, D. 2007. Biotin sensing: universal influence of biotin status on transcripstion. Annu. Rev. Genet. 41. 443-464.

[25] Copley, R.R., G.J. Barton. 1994. A structural analysis of phosphate and sulphate binding sites in proteins. Estimation of propensities for binding and conservation of phosphate binding sites. J. Mol. Biol. 242. 321-329.

[26] Sarkar, S., A.K. Hopper. 1998. tRNA Nuclear Export in Saccharomyces cerevisiae: In Situ Hybridization Analysis. Mol. Biol. Cell. 9(11). 3041-3055. 